\title{
Patient reported outcomes: integration into clinical practices
}

\author{
Nakul Valsangkar, Felix Fernandez, Onkar Khullar \\ Division of Cardiothoracic Surgery, Emory University School of Medicine, Atlanta, GA, USA \\ Contributions: (I) Conception and design: O Khullar, F Fernandez; (II) Administrative support: O Khullar, F Fernandez; (III) Provision of study \\ materials or patients: All authors; (IV) Collection and assembly of data: All authors; (V) Data analysis and interpretation: All authors; (VI) Manuscript \\ writing: All authors; (VII) Final approval of manuscript: All authors. \\ Correspondence to: Onkar Khullar, MD. Division of Cardiothoracic Surgery, Emory University School of Medicine, 550 Peachtree Street, 6th Floor, \\ Atlanta, GA 30308, USA. Email: onkar.vohra.khullar@emory.edu.
}

\begin{abstract}
Patient-centered care is a growing focus of research and modern surgical practice. To this end, there has been an ever-increasing utilization of patient reported outcomes (PRO) and health-related quality of life metrics (HR-QOL) in thoracic surgery research. Here we describe reasons and methods for integration of PRO measurement into routine thoracic surgical practice, commonly utilized PRO measurement instruments, and several examples of successful integration.
\end{abstract}

Keywords: Patient reported outcomes (PRO); European Organization for Research and Treatment of Cancer
(EORTC); Patient Reported Outcomes Measurement Information System (PROMIS); quality of life (QOL);
health-related quality of life (HR-QOL); patient centered care

Submitted Mar 10, 2020. Accepted for publication Apr 20, 2020.

doi: $10.21037 /$ jtd.2020.03.91

View this article at: http://dx.doi.org/10.21037/jtd.2020.03.91

\section{Introduction}

Patient-centered care is a growing focus of research and modern surgical practice. Patient reported outcomes (PRO) have been demonstrated to improve physician-patient communication, help in addressing patient symptoms, and improve patients' quality of life. PROs have also been identified as an independent prognostic marker of survival in both esophageal and lung cancer (1-5). To this end, there has been increasing utilization of PRO and health-related quality of life metrics (HR-QOL) in thoracic surgery research. Such studies range from small single institution retrospective studies to large multi-institutional randomized clinical trials $(6,7)$. In well instituted programs, PROs can help clinicians monitor adverse events in real time, change chemotherapy regimens faster, reprioritize timing of clinic visits, optimize supportive pharmacotherapy, and more effectively perform recurrence monitoring.

PROs have been pushed to the forefront of comparative effectiveness research (CER) and outcomes measurement. National organizations, such as the Center for Medicare and Medicaid (CMS), National Institutes of Health (NIH), and the American College of Chest Physicians (ACP), have endorsed the collection and analysis of PRO measures in outcomes and performance measurement (8). As part of its quality initiative, CMS has specific programs (value-based purchasing, and hospital readmissions reduction) where quality of care, readmission data, and physician performance are taken into consideration while calculating payment adjust factors for reimbursement (9-11). As a result, PRO measures increasingly factor into medical and system-wide financial decision making. The implementation of PRO into clinical practice is now a high priority and an unprecedented opportunity.

The application of PRO into clinical practice however has several challenges. These include the need of an electronic platform and IT support for PRO measurement, patient and provider education around implementation and utilization, clinical provider buy-in and prioritization, and ultimately-costs (2). Furthermore, there is also no clear consensus on the most effective methods to collect, and analyze these data, and consequently to impart real time change in patient management.

The objective of this manuscript is to: (I) compare 
Table 1 Commonly used patient reported outcome (PRO) measurement instruments

General global health PRO instruments
Depression, Anxiety, and Stress Scale 21 (DASS-21)
Edmonton Symptom Assessment Score (ESAS)
MD Anderson Symptom Inventory
Medical Outcomes Study Short Form 36 (SF 36)
Nottingham Health Profile
Patient-Reported Outcomes Measurement Information System
(PROMIS)
Rotterdam Symptom Checklist
Disease and symptom specific PRO instruments
Dyspnea Index
Dysfunction After Upper Gastrointestinal Surgery (DAUGS-20)
EuroQual quality of life questionnaire (EQ-5D)
European Organization for Research and Treatment of Cancer
(EORTC) Modules
Quality of Life Questionnaire Core 30 (QLQ C-30)
QLQ Lung Module 13 (LC13)
QLQ Esophagus Module 18 (OES18)
Functional Assessment of Cancer Therapy (FACT) Oncologic
and Organ Specific Modules
Lung Module (FACT-L)
Esophagus Module (FACT-E)
Prient health questionnaire (PHQ-2)
Shortity, health and labor questionnaire)

the most frequently used PRO instruments in thoracic oncology; (II) briefly review the current literature on the collection and analysis of PRO data in thoracic surgery; and (III) to discuss successful applications of PRO into clinical practices and the lessons learned from these implementations.

\section{Common PRO instruments}

There are a variety of PRO instruments available for use in clinical practice. In fact, this is one of the greatest impediments to widespread utilization and integration. These instruments range from generic questionnaires focusing on global health universal to all patients, to disease- or even symptoms-specific questionnaires applicable to a small subset of patients (Table 1). One of the greatest challenges with PRO research and utilization is this variety of instruments, many of which are scored differently, making direct comparisons and interpretation difficult.

The most commonly used instruments evaluate overall quality of life whereas more specific forms are available to evaluate patients with cancer, such as those with thoracic malignancies. One of the most frequently utilized instruments is the RAND Medical Outcomes Study 36Item Short Form Health Survey (SF-36) and its short version the SF-20, developed as part of a multi-institutional study to explain variations in patient outcome (12-14). It is widely used in many research studies, clinical practice, as well as in macroscopic applications such as policy and larger population-based surveys. While this measure was not specifically designed for thoracic surgery or oncology patients, it is widely applicable and has many uses.

Several well-validated cancer-specific questionnaires are available for use as well (Table 1). For example, the Rotterdam symptom checklist (RSCL) was designed for oncology patients and measures both the physical and psychological aspects of the quality of life $(1,15)$. In a randomized control trial comparing transthoracic and transhiatal esophagectomy; in addition to tumor stage postoperative social functioning and patient reported activity level, measured with the SF-20 and the RSCL, were found to be independent predictors of survival (16).

Two other commonly used instruments are the Functional Assessment of Cancer Therapy (FACT-G) questionnaire and the EORTC QOL questionnaires. Each of these have esophagus and lung disease specific modules. The original FACT tool has four domains, physical, social and family, emotional, and functional well-being; and was developed in breast and colorectal cancer patients. The European Organization for Research and Treatment of Cancer (EORTC) QOL Questionnaire Core 30 (EORTC QLQ-C30) is a 30-point questionnaire and includes, symptom scales, functional scales, as well as scales for global health. Many comparative effectiveness research studies use one or the other tool. Undoubtedly, these tools are among the most commonly utilized in thoracic surgical research (17).

An important consideration remains that the results of these questionnaires are not always in agreement with each other. One study compared the EORTC QLQOES18 with the FACT-E in patients with esophageal cancer with mixed findings (17). The results indicated a 
good correlation between the two instruments regarding physical function scores. However, the correlation between the social functioning scale of the FACTG and the EORTC QLQ-C30 was poor. The authors concluded that these scales may not be interchangeable and should be used in accordance with the desired outcome of the study. In the end, while many of these instruments may sound similar, the results can be somewhat different depending on the instrument used. For that reason, it is important to select a carefully validated PRO measure, that is applicable to each clinician's outcome of interest.

In this regard, the Patient Reported Outcomes Measurement Information System (PROMIS), may an ideally suited platform. PROMIS is an NIH-sponsored, prospectively validated $\mathrm{PRO}$ measurement system that was developed to be applicable to the general population with a variety of modules/instruments which are specific to a health domain, rather than a disease $(18,19)$. Our group has published an observational study of over 120 lung cancer surgery patients who were administered a PRO questionnaire consisting of 12 PROMIS instruments, integrating these results with our institutional Society of Thoracic Surgeons database (20). PROMIS has the advantage of generating an easily interpretable and comparable result, standardized to a T-score distribution based on test results from the general US population. It has been validated in cancer patients and can easily be administered electronically (1,19-21). However, it has not been widely utilized in thoracic surgical literature to date.

\section{How are PROs collected?}

The methodologies to collect and analyze PRO are as varied as the instruments and the type of clinical practices that currently employ them. For example, PRO can be collected via in-person surveys that build into clinic workflows, or they can be administered either via telephone or mail either before or after the patient's encounter. They can be paper based or electronic forms. Electronic administration is the preferred method for most given the increased flexibility it affords. Electronic surveys, particularly if web-based, can be completed at home, in the office, or even after a clinic visit depending on the instrument utilized, the questions of interest, and the platform for administration. The patients can also be emailed links for online surveys or provided with the survey during a clinic visit, depending on which integrates into clinical workflow most easily.

Ultimately the data is filtered through a common IT system and is associated with the patients' medical records. Depending on the type of scoring system used this can either be a summary score or scores in several domains. Many of the commonly utilized EMR systems allow for real-time integration of PRO results into the medical records, a step necessary for utilizing PRO results in day to day clinical management of patients. Lastly, the timing of collection can also be discrete time points before and after surgery or at equal intervals to determine changes in specific symptoms. If there is a frequent reporting of symptoms, it is usually performed via a web-based form, and there should be a robust system to escalate the symptom to clinicians who can then take actions based on this reported data (22).

\section{Setting standards in the collection and analysis of PRO data}

Although there are a large number of PRO instruments, and they are frequently collected as part of secondary end points in clinical trials, there are no existing standard in the collection and interpretation of PRO data. This makes it difficult to compare conclusions drawn from different studies. There are recommendations to improve reporting of PROs in study protocols [Standard Protocol Items: Recommendations for Interventional Trials-PRO extension (SPIRIT-PRO)] as well as in publications [Consolidated Standards of Reporting Trials Statement-PRO extension (CONSORT-PRO)] (23). These recommendations are geared towards the uniform reporting and analysis of PRO across different clinical trials and research studies so they can be meaningfully compared to effect change in treatment choices, health care policy and improve patient safety. In order to consolidate all of these under one umbrella, the global Setting International Standards in Analyzing Patient-Reported Outcomes and Quality of Life Endpoints Data (SISAQOL) Consortium was initiated, which includes a wide variety of stake holders in patient-centered care including: physicians, industry representatives, biostatisticians, and regulatory bodies (23).

\section{Clinical integration of PROs}

The application of PRO to clinical practice in Europe and North America has seen several successful yet somewhat different approaches. The American Society of Clinical Oncology in its 2018 meeting dedicated an entire session to the implementation of PROs in clinical practice, as individual institutions and health system experiences 
have provided unique insights (22). Currently, with the widespread prevalence of the electronic medical record, most PRO applications begin with registering the patient into an internet-based data collection system and providing the patient a computer kiosk, touch-screen based tablet, or online web portal access to be able to complete the PRO questionnaire electronically. Real-time integration of the results into the electronic health record is then provided to the clinical team in order to incorporate the relevant patient responses into the visit. At a predetermined set point, the patient and health care team can also receive a summary of previous visits/results in order to identify trends in symptoms or deviations from expectations. This can then be used to assist clinical decision making and patient-centered care.

As mentioned, our preferred instrument is PROMIS. PROMIS is an NIH-sponsored PRO instrument that consists of multiple provider-chosen modules which are symptoms specific, rather than disease specific. It utilizes large-bank question items and computerized adaptive testing in order to administer an efficient, user-friendly interface. We have previously published a series of to evaluate 127 patients undergoing lung cancer resection over 18 months using a composite questionnaire that included three key PROMIS domains. These patients were provided touch screen tablet devices during their clinic appointments at three predetermined set points (pre-operative, 1-month post-operative, and 6-month post-operative visits). This study found a decline in patient-reported pain, fatigue, sleep deprivation, and physical function at 1-month post-operative visit, and a near return to baseline at 6 months (20). Subsequent, to date unpublished, results have shown significant correlation between PRO and pulmonary function, as well as with postoperative surgical outcomes.

PRO systems can also be applied to a population level. For example, in Canada, Cancer Care Ontario (CCO) is responsible for multisystem planning, deployment of information technology systems, and collection and analysis of PRO data utilizing the Edmonton Symptom Assessment Scale (ESAS). Patients who are registered with CCO and undergoing therapy for any form of cancer, complete the PRO questionnaire at a computer kiosk prior to their appointment on a monthly basis. The results of their survey, and previous cumulative results, are provided to the treating clinical team prior to the visit. Implementation of this system has led to better patient symptom management, and clinician knowledge of the patients' symptoms. This in turn was anticipated to minimize otherwise unnecessary emergency room visits. A pilot study of this system reported a significant association between the implementation of this system on a province wide level with increased documentation of patient symptoms, and decreased use of acute care services by the patient $(22,24,25)$.

Patients undergoing chemotherapy and in the postoperative setting often underestimate the degree of some symptoms and are not sure of the appropriate responses. Another country wide iteration of a PRO system includes the deployment of a system that is available online and is able to provide real time feedback for patients at all times. These considerations were used to develop the eRAPID (Electronic patient self-Reporting of Adverse-events: Patient Information and aDvice) system in the UK. This is a 5-year multi-institution research program funded by the NIH. This system involves a robust IT system in the backend of a website where patients report their symptoms remotely from home, and get immediate tailored recommendations regarding their symptoms. These reported symptoms are graded by the system from most severe (A) through no symptoms (D). The system also generates an email for the primary clinical team, and this data is transferred to the electronic medical record. A pilot study indicated that $90 \%$ of patients were found to be in the non-emergent categories of B-three or more moderately severe symptoms; with the recommendation: contact medical team when convenient, or C-mild symptoms, recommendation: follow selfmanagement advice. Implementation of this system resulted in saved acute care visits, decreased costs, and improved patient satisfaction $(22,26)$.

More frequent reporting of symptoms and accurate tracking of these symptoms by health care professionals has also been studied in the US. A recent large randomized clinical trial at Memorial Sloan Kettering Cancer Center (MSKCC) that enrolled 766 patients with advanced solid tumors receiving outpatient chemotherapy, demonstrated that when PROs were routinely collected, tracked, and acted upon using a dedicated web-based system, patients experienced improved health care related QOL, reduced utilization of emergency services and improved overall survival. The system at MSKCC used the web-based Symptom Tracking and Reporting (STAR) platform which allowed patients to $\log 12$ chemotherapy associated side effects on a weekly basis, and nurses received alerts when there was an escalation in the symptom grade. This triggered a notification to the patients care team 


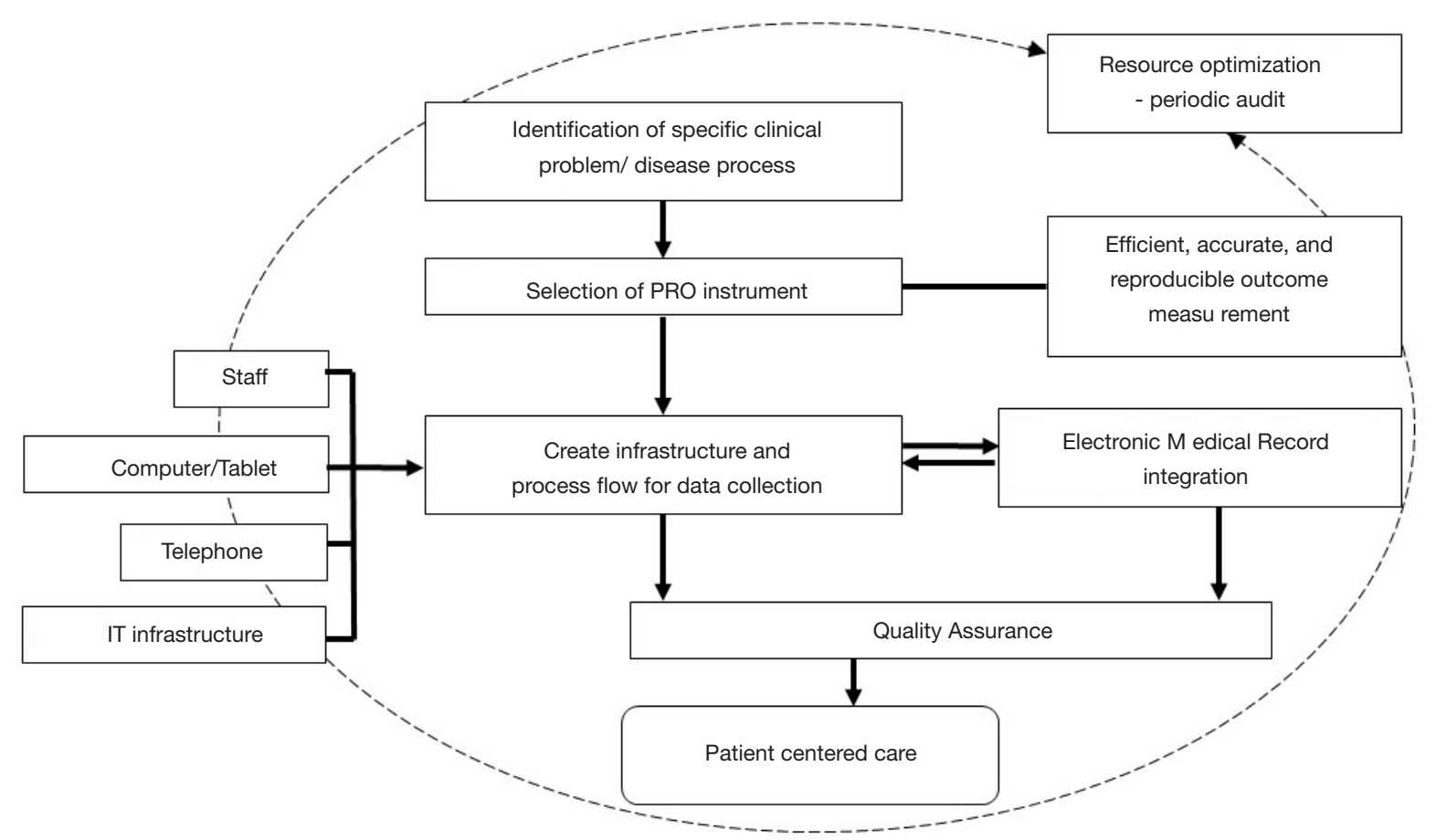

Figure 1 The key steps for the application of PRO in clinical practice include; identification of a specific clinical problem, selection of an appropriate PRO instrument, creating a thoughtful process flow, identification of ways to integrate the PRO data into the electronic medical record, and ultimately optimally using all available resources with a high level of quality control. PRO, patient reported outcome.

and additional actions depending on the symptom. The patients in the study arm had a median overall survival of 31.2 months compared with 26 months for those in the control $\mathrm{arm}$. This was the single largest documented improvement in median overall survival in that group of patients $(27,28)$.

These studies suggest that the transition from the development and validation of a PRO instrument to the provision of patient centered care follows a set of wellplanned steps such as indicated in Figure 1. The following six steps were identified as the cornerstone to a successful application of PRO in clinical practice.

(I) Identification of a specific clinical problem, disease process, and outcome measure that is desired. Depending on the study population this may be a particular symptom, or symptoms from a specific domain such as social adjustment, pain, or physical function.

(II) Selection of the appropriate PRO instrument that most accurately measures this outcome measure in an efficient, accurate, and reproducible fashion.
(III) Process flow: creation of the infrastructure and process flow for data collection. This includes additional nursing staff, touch screen devices, computer kiosks, telephone lines and supporting IT infrastructure, depending on the size of the project and implementation.

(IV) Electronic medical record: identification of the frequency of data collection, sending patient reminders, and generation of a system-automated or manual to integrate the $\mathrm{PRO}$ data into the electronic medical record.

(V) Quality assurance: frequent audit of patient charts with high PRO scores to identify if there were any actionable items generated, review the clinical decision making and see if there was any improvement in the patient symptomatology. If not, ensure that there is a feedback mechanism to the stakeholders for quality assurance. The presence of a specific institutional or local health care provider who champions this quality assurance 
process is important.

(VI) Resource optimization: identify processes that can be either automated or queued for the next clinic encounter to increase efficiency in the system. There should also be mechanisms designed to avoid repetitive $\mathrm{PRO}$ questionnaires similar to what patients may have recently filled for another clinic for example-oncology and surgical clinics having similar PRO questionnaires for the post-operative patient.

\section{Conclusions}

Patient-centered care is ultimately dependent on the timely collection of patient-reported data using a well-designed online platform that easily integrates into the electronic medical record and generates actionable items that are expediently resolved at the appropriate level of care. For all these steps to work in unison, there needs to be robust IT support, highly motivated ancillary support staff, well trained nursing support, and a quality driven process that has feedback loops for continuous improvement. The successful implementation of this process is marked with continually improving patient satisfaction, and symptom scores across multiple PRO domains along with improvements in survival. This ultimately reduces the utilization of unnecessary acute care services, and decreasing readmission rates, helping to provide the best possible care to patients with the least financial burden to the health care system.

\section{Acknowledgments}

Funding: This project was supported by grant from the American Association for Thoracic Surgery Graham Foundation Cardiothoracic Surgical Investigators Program.

\section{Footnote}

Provenance and Peer Review: This article was commissioned by the Guest Editor (Peter J. Kneuertz) for the series "Patient reported Outcomes in Thoracic Surgery: A new Frontier" published in Fournal of Thoracic Disease. The article has undergone external peer review.

Conflicts of Interest: All authors have completed the ICMJE uniform disclosure form (available at http://dx.doi. org/10.21037/jtd.2020.03.91). The series "Patient reported
Outcomes in Thoracic Surgery: A new Frontier" was commissioned by the editorial office without any funding or sponsorship. The other authors have no other conflicts of interest to declare.

Ethical Statement: The authors are accountable for all aspects of the work in ensuring that questions related to the accuracy or integrity of any part of the work are appropriately investigated and resolved.

Open Access Statement: This is an Open Access article distributed in accordance with the Creative Commons Attribution-NonCommercial-NoDerivs 4.0 International License (CC BY-NC-ND 4.0), which permits the noncommercial replication and distribution of the article with the strict proviso that no changes or edits are made and the original work is properly cited (including links to both the formal publication through the relevant DOI and the license). See: https://creativecommons.org/licenses/by-nc-nd/4.0/.

\section{References}

1. Subramanian M, Kozower BD, Brown LM, et al. PatientReported Outcomes in Cardiothoracic Surgery. Ann Thorac Surg 2019;107:294-301.

2. Basch E, Snyder C. Overcoming barriers to integrating patient-reported outcomes in clinical practice and electronic health records. Ann Oncol 2017;28:2332-3.

3. Langendijk H, Aaronson NK, de Jong JM, et al. The prognostic impact of quality of life assessed with the EORTC QLQ-C30 in inoperable non-small cell lung carcinoma treated with radiotherapy. Radiother Oncol 2000;55:19-25.

4. Movsas B, Moughan J, Sarna L, et al. Quality of life supersedes the classic prognosticators for long-term survival in locally advanced non-small-cell lung cancer: an analysis of RTOG 9801. J Clin Oncol 2009;27:5816-22.

5. Möller A, Sartipy U. Associations between changes in quality of life and survival after lung cancer surgery. J Thorac Oncol 2012;7:183-7.

6. Fernando HC, Landreneau RJ, Mandrekar SJ, et al. Analysis of longitudinal quality-of-life data in high-risk operable patients with lung cancer: Results from the ACOSOG Z4032 (Alliance) multicenter randomized trial. J Thorac Cardiovasc Surg 2015;149:718-25.

7. Blazeby JM, Kavadas V, Vickery CW, et al. A prospective comparison of quality of life measures for patients with esophageal cancer. Qual Life Res 2005;14:387-93. 
8. Lipscomb J, Gotay CC, Snyder CF. Patient-reported Outcomes in Cancer: A Review of Recent Research and Policy Initiatives. CA Cancer J Clin 2007;57:278-300.

9. Stacy KM. Hospital Value-Based Purchasing: Part 2, Implications. AACN Adv Crit Care 2017;28:16-20.

10. Gupta A, Fonarow GC. The Hospital Readmissions Reduction Program-learning from failure of a healthcare policy. Eur J Heart Fail 2018;20(8):1169-1174.

11. Hospital Readmissions Reduction Program (HRRP). Available online: https://www.cms.gov/Medicare/ Medicare-Fee-for-Service-Payment/AcuteInpatientPPS/ Readmissions-Reduction-Program. Accessed August 3, 2020.

12. 36-Item Short Form Survey (SF-36). Available online: https://www.rand.org/health-care/surveys_tools/mos/36item-short-form.html. Accessed September 3, 2020.

13. Hays RD, Sherbourne CD, Mazel RM. The rand 36-item health survey 1.0. Health Econ 1993;2:217-27.

14. DiMatteo MR, Sherbourne CD, Hays RD, et al. Physicians' characteristics influence patients' adherence to medical treatment: results from the Medical Outcomes Study. Health Psychol 1993;12:93-102.

15. Khullar O V., Fernandez FG. Patient-Reported Outcomes in Thoracic Surgery. Thorac Surg Clin 2017;27:279-90.

16. van Heijl M, Sprangers MAG, De Boer AGEM, et al. Preoperative and early postoperative quality of life predict survival in potentially curable patients with esophageal cancer. Ann Surg Oncol 2010;17:23-30.

17. Straatman J, Joosten PJM, Terwee CB, et al. Systematic review of patient-reported outcome measures in the surgical treatment of patients with esophageal cancer. Dis Esophagus 2016;29:760-72.

18. Gershon RC, Rothrock N, Hanrahan R, et al. The use of PROMIS and assessment center to deliver patientreported outcome measures in clinical research. J Appl Meas 2010;11:304-14.

19. Jones RS, Stukenborg GJ. Patient-Reported Outcomes Measurement Information System (PROMIS) Use

Cite this article as: Valsangkar N, Fernandez F, Khullar O. Patient reported outcomes: integration into clinical practices. J Thorac Dis 2020;12(11):6940-6946. doi: 10.21037/jtd.2020.03.91 in Surgical Care: A Scoping Study. J Am Coll Surg 2017;224:245-254.e1.

20. Khullar OV, Rajaei MH, Force SD, et al. Pilot Study to Integrate Patient Reported Outcomes After Lung Cancer Operations Into The Society of Thoracic Surgeons Database. Ann Thorac Surg 2017;104:245-53.

21. Hirpara DH, Gupta V, Brown L, et al. Patient-reported outcomes in lung and esophageal cancer. J Thorac Dis 2019;11:S509-14.

22. Basch E, Barbera L, Kerrigan CL, et al. Implementation of Patient-Reported Outcomes in Routine Medical Care. Am Soc Clin Oncol Educ book Am Soc Clin Oncol Annu Meet 2018;38:122-34.

23. Coens C, Pe M, Dueck AC, et al. International standards for the analysis of quality-of-life and patient-reported outcome endpoints in cancer randomised controlled trials: recommendations of the SISAQOL Consortium. Lancet Oncol 2020;21:e83-96.

24. Dudgeon D, Vaitonis V, Seow H, et al. Ontario, Canada: using networks to integrate palliative care province-wide. J Pain Symptom Manage 2007;33:640-4.

25. Dudgeon DJ, Knott C, Eichholz M, et al. Palliative Care Integration Project (PCIP) quality improvement strategy evaluation. J Pain Symptom Manage 2008;35:573-82.

26. Absolom K, Holch P, Warrington L, et al. Electronic patient self-Reporting of Adverse-events: Patient Information and aDvice (eRAPID): a randomised controlled trial in systemic cancer treatment. BMC Cancer 2017;17:318.

27. Basch E, Deal AM, Kris MG, et al. Symptom Monitoring With Patient-Reported Outcomes During Routine Cancer Treatment: A Randomized Controlled Trial. J Clin Oncol 2016;34:557-65.

28. Basch E, Deal AM, Dueck AC, et al. Overall Survival Results of a Trial Assessing Patient-Reported Outcomes for Symptom Monitoring During Routine Cancer Treatment. JAMA 2017;318:197-8. 